\title{
Correction to: Early versus late intramedullary nailing for traumatic femur fracture management: meta-analysis
}

\author{
Ayman El-Menyar ${ }^{1,2,3 *}$, Mohammed Muneer ${ }^{4}$, David Samson ${ }^{1}$, Hassan Al-Thani ${ }^{5}$, Ahmad Alobaidi ${ }^{6}$,
} Paul Mussleman ${ }^{7}$ and Rifat Latifi ${ }^{8}$

\section{Correction}

Following the publication of this article [1], the authors reported that they had submitted an incorrect version of Figs. 2, 3 and 4. They apologize for this error and the correct versions of Figs. 2, 3 and 4 with captions have been included in this Correction. There is a typographical error in the following sentence: Two retrospective cohort studies reported results on any and all complications [23, 30]. The correct version of this sentence is: Two retrospective cohort studies reported results on any complication [24, 34].

\footnotetext{
Author details

'Department of Surgery Clinical Research Unit, Westchester Medical Center Health Network, Valhalla, NY, USA. ${ }^{2}$ Trauma Surgery, Clinical Research, Hamad General Hospital, Doha, Qatar. ${ }^{3}$ Clinical Medicine, Weill Cornell Medical School, Doha, Qatar. ${ }^{4}$ Department of Surgery, Hamad General Hospital, Doha, Qatar. ${ }^{5}$ Department of Surgery, Trauma and Vascular Surgery, Hamad General Hospital, Doha, Qatar. ${ }^{6}$ Department of Surgery, Orthopedic Surgery, Al Wakrah Hospital, Doha, Qatar. ${ }^{7}$ Distributed eLibrary, Weill Cornell Medical School, Doha, Qatar. ${ }^{8}$ Department of Surgery, Westchester Medical Center Health Network and New York Medical College, Valhalla, NY, USA.
}

Received: 5 July 2018 Accepted: 6 July 2018

Published online: 24 July 2018

\section{Reference}

1. El-Menyar, et al. Early versus late intramedullary nailing for traumatic femur fracture management: meta-analysis. J Orthop Surg Res. 2018;13:160. https://doi.org/10.1186/s13018-018-0856-4.

\footnotetext{
* Correspondence: aymanco65@yahoo.com

1Department of Surgery Clinical Research Unit, Westchester Medical Center Health Network, Valhalla, NY, USA

${ }^{2}$ Trauma Surgery, Clinical Research, Hamad General Hospital, Doha, Qatar

Full list of author information is available at the end of the article
}

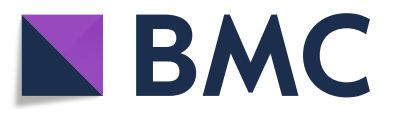

(c) The Author(s). 2018 Open Access This article is distributed under the terms of the Creative Commons Attribution 4.0 International License (http://creativecommons.org/licenses/by/4.0/), which permits unrestricted use, distribution, and reproduction in any medium, provided you give appropriate credit to the original author(s) and the source, provide a link to the Creative Commons license, and indicate if changes were made. The Creative Commons Public Domain Dedication waiver (http://creativecommons.org/publicdomain/zero/1.0/) applies to the data made available in this article, unless otherwise stated. 
a) Pulmonary complications

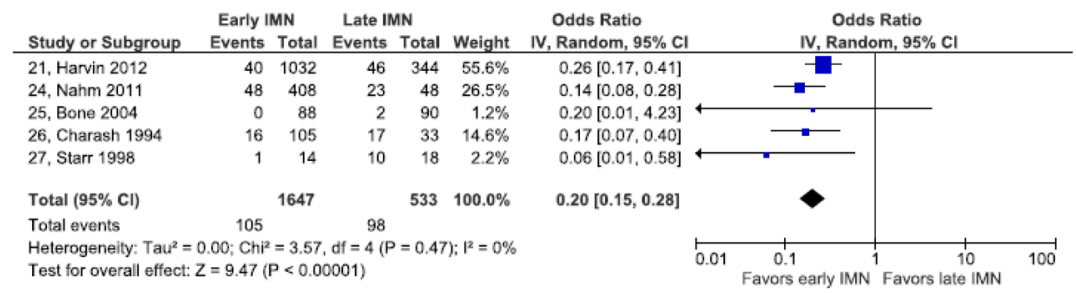

b) Acute respiratory distress syndrome

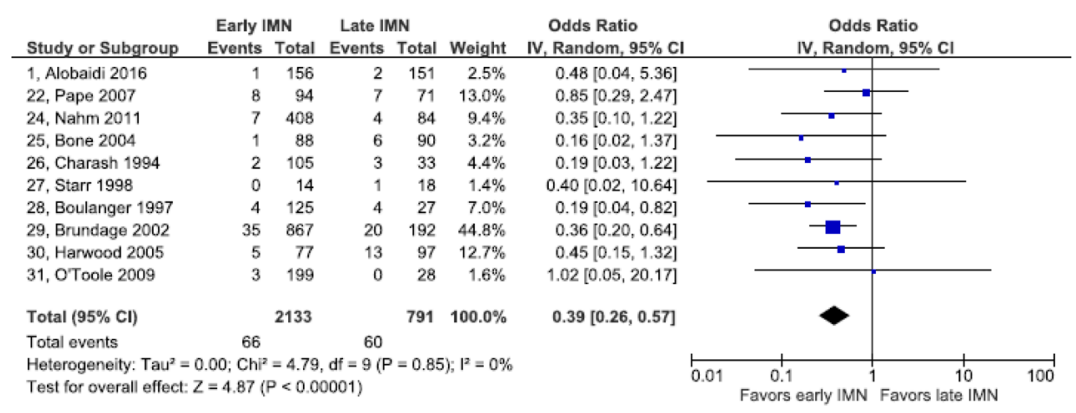

C) Fat embolism

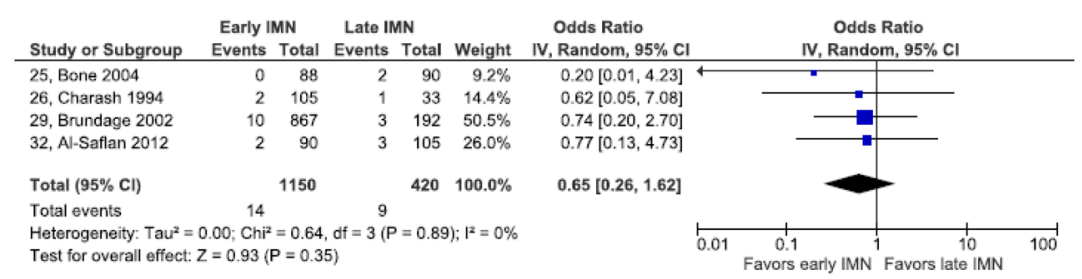

d) Pulmonary embolism

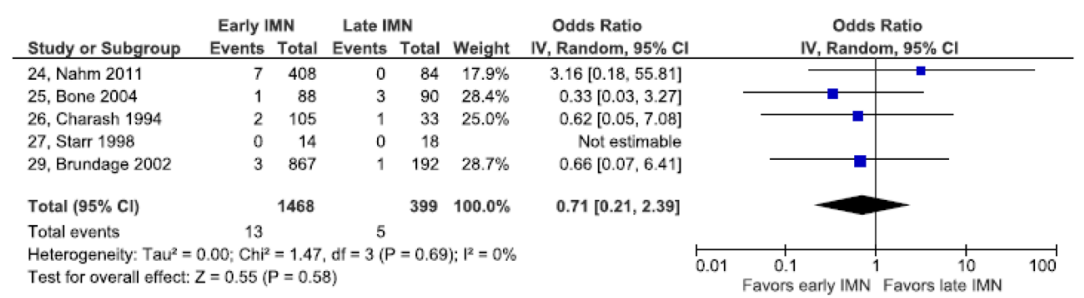

e) Pneumonia

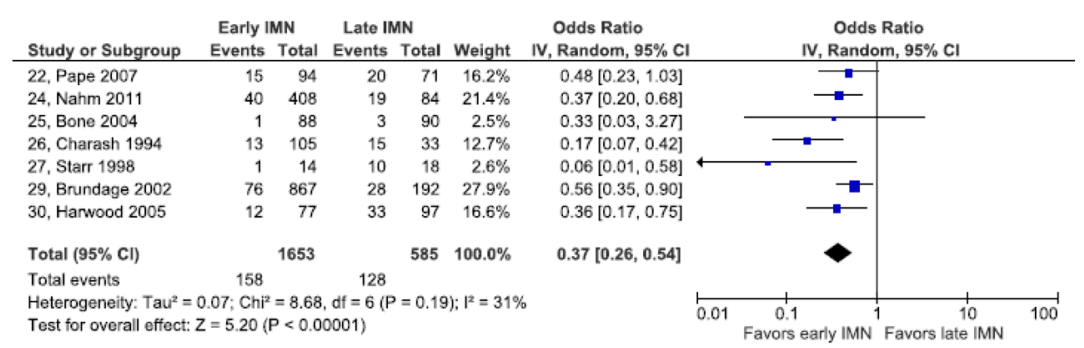

Fig. 2 Forest plot of pulmonary complications. a Pulmonary complications. b Acute respiratory distress syndrome. c Fat embolism. d pulmonary embolism. e Pneumonia 
a) Decubitus ulcers

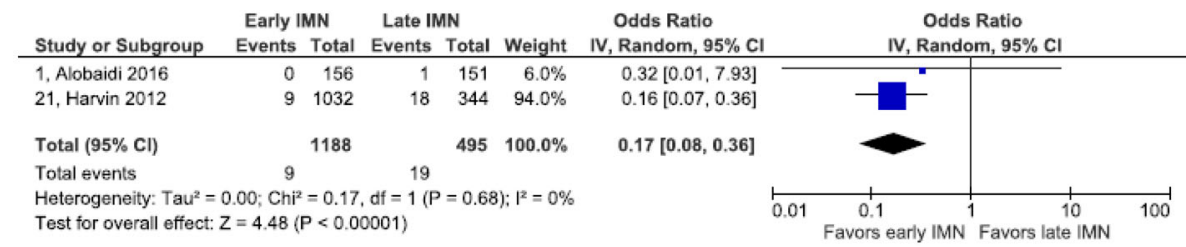

b) Wound infection

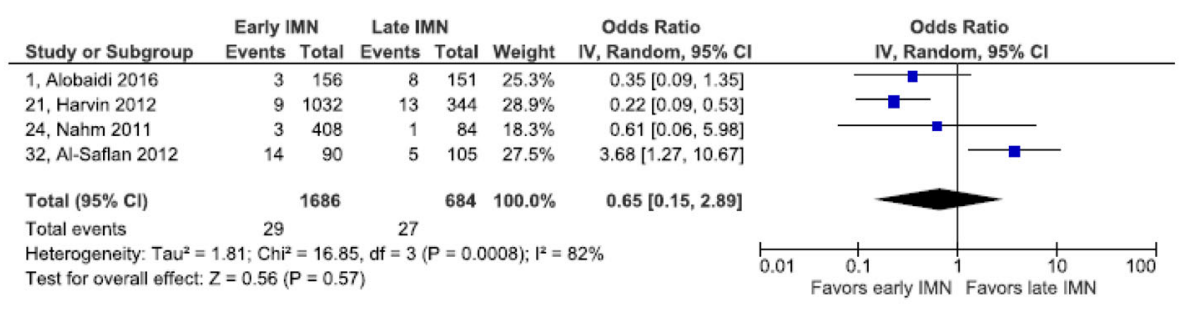

c) Sepsis

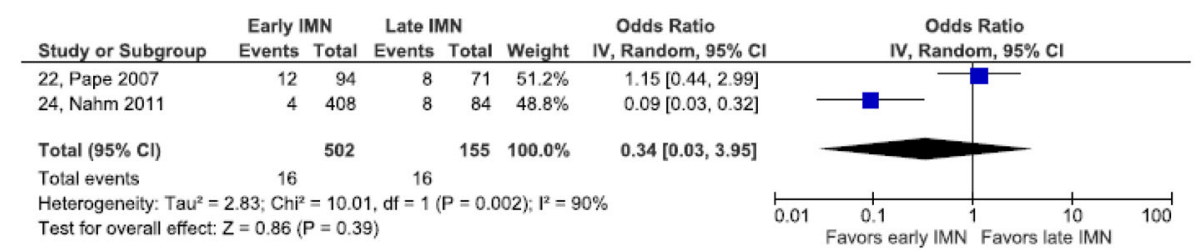

d) Deep vein thrombosis

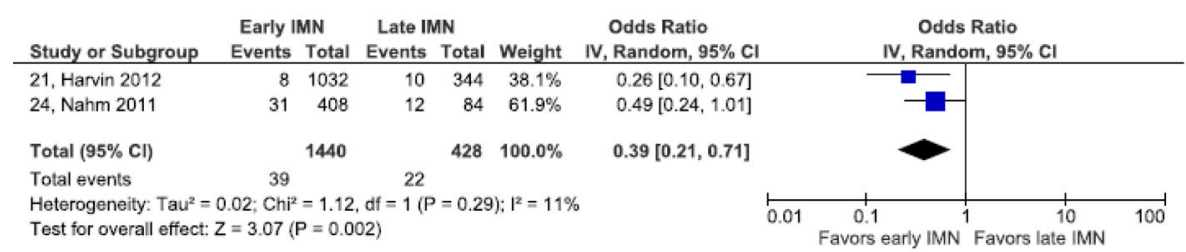

Fig. 3 Forest plots of cutaneous, infectious and venous complications: a decubitus ulcers. b wound infection. c sepsis. $\mathbf{d}$ deep vein thrombosis 
a) Any complication

\begin{tabular}{|c|c|c|c|c|c|c|c|c|c|c|}
\hline Study or Subgroup & \multicolumn{2}{|c|}{ Early IMN } & \multicolumn{2}{|c|}{ Late IMN } & Weight & $\begin{array}{l}\text { Odds Ratio } \\
\text { IV, Random, } 95 \% \mathrm{Cl}\end{array}$ & \multicolumn{4}{|c|}{$\begin{array}{c}\text { Odds Ratio } \\
\text { IV, Random, 95\% Cl }\end{array}$} \\
\hline 24, Nahm 2011 & 77 & 408 & 36 & 84 & $50.2 \%$ & $0.31[0.19,0.51]$ & & -1 & & \\
\hline 32, Al-Saflan 2012 & 54 & 90 & 23 & 105 & $49.8 \%$ & $5.35[2.86,10.00]$ & & & $\rightarrow-$ & \\
\hline Total $(95 \% \mathrm{Cl})$ & & 498 & & 189 & $100.0 \%$ & $1.28[0.08,20.84]$ & & & & \\
\hline Total events & 131 & & 59 & & & & & & & \\
\hline $\begin{array}{l}\text { Heterogeneity: } \mathrm{Tau}^{2} \\
\text { Test for overall effect }\end{array}$ & $\begin{array}{l}3.97 ; \mathrm{Chi}^{2} \\
Z=0.17(F\end{array}$ & $=48.6$ & 7. $d f=1($ & $(P<0.0$ & 00001): $I^{2}$ & $=98 \%$ & 0.01 & $\begin{array}{l}0.1 \\
\text { vors early IMI }\end{array}$ & 100 & 100 \\
\hline
\end{tabular}

b) Multiorgan failure

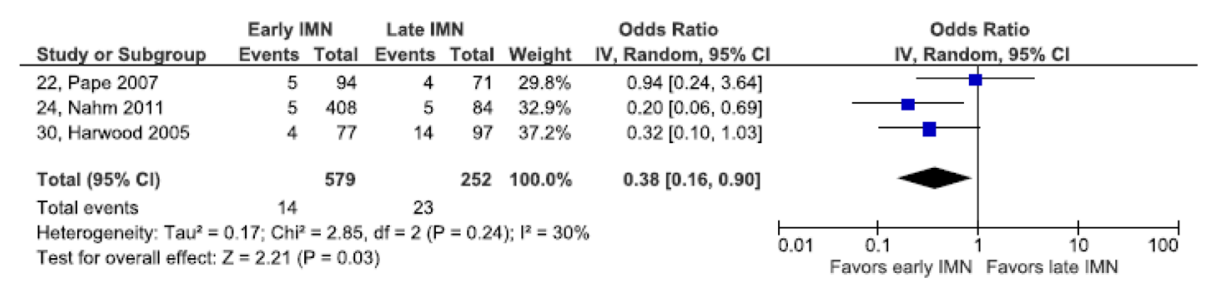

c) Mortality

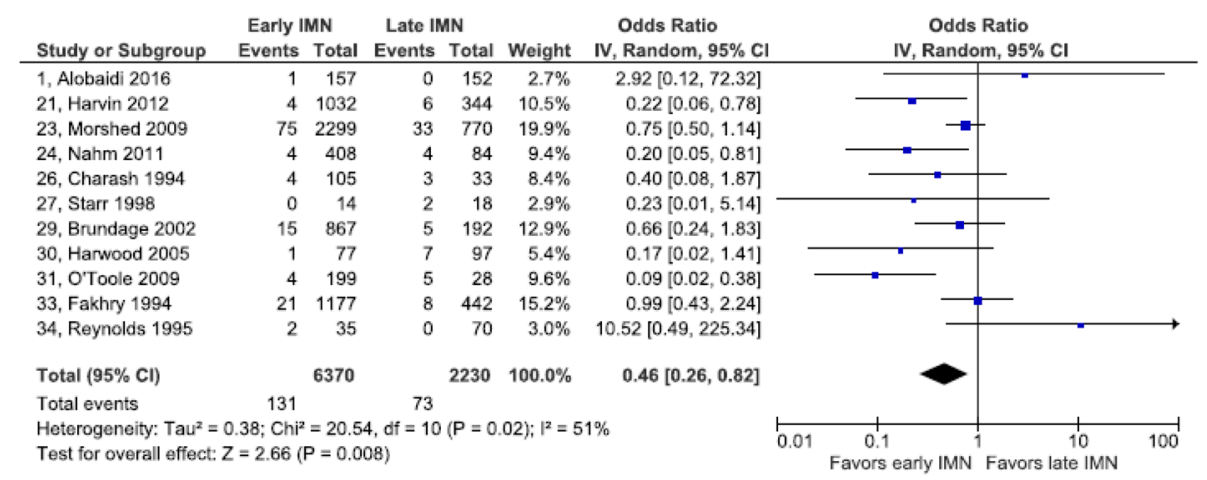

Fig. 4 Forest plots of other complications: a. Any complication. b multiorgan failure c. mortality 